\title{
Quaderni
}

QUADERNI Communication, technologies, pouvoir

97 | Automne 2018

Néo-libéralisme(s). Réseaux et formes des

mobilisations en France

\section{"Les idées ont des conséquences" : la genèse internationale des think tanks néo-libéraux français}

\section{Kevin Brookes}

\section{Édition électronique}

URL : http://journals.openedition.org/quaderni/1277

DOI : 10.4000/quaderni.1277

ISSN : 2105-2956

\section{Éditeur}

Les éditions de la Maison des sciences de l'Homme

\section{Édition imprimée}

Date de publication : 5 octobre 2018

Pagination : 35-55

\section{Référence électronique}

Kevin Brookes, «"Les idées ont des conséquences" : la genèse internationale des think tanks néo-

libéraux français », Quaderni [En ligne], 97 | Automne 2018, mis en ligne le 05 octobre 2020, consulté le 05 janvier 2021. URL : http://journals.openedition.org/quaderni/1277 ; DOI : https://doi.org/10.4000/ quaderni. 1277 


\section{$D$ ossier}

\section{"Les idées ont des conséquences" :}

la genèse internationale

des think tanks néo-libéraux français

\section{Kevin Brookes}

Université Grenoble Alpes Science Po Grenoble / PACTE
Le philosophe et économiste Friedrich Hayek a proposé en 1949 une stratégie pour diffuser les idées néo-libérales et contrer intellectuellement le socialisme. Dans sa perspective idéaliste, il convenait d'abord de convaincre les intellectuels afin de changer le « climat d'opinion » et par ce truchement, la politique de demain. Il accordait ainsi à la figure de l'intellectuel une place centrale :

«En matière d'événements ou d'idées, il n'est pas grand-chose que l'homme ordinaire apprenne autrement que par l'intermédiaire de [la classe des intellectuels]. [...] Ce sont les intellectuels qui décident quelles analyses et quelles opinions nous parviendront, quels faits sont suffisamment importants pour nous être révélés, et sous quelle forme et selon quel point de vue ils nous seront présentés. C'est principalement à leur décision que nous devons d'être (ou de ne pas être) informés des résultats des travaux des experts ou des penseurs $^{1}$. \>

Depuis l'écriture de cet article, la circulation transnationale du néo-libéralisme a été le fruit d'un long de travail de dissémination opéré à travers des intellectuels et organisations internationales partageant cette même perspective valorisant le combat d'idées. Au-delà des formes savantes de diffusion du néo-libéralisme qui ont fait l'objet de nombreuses études, notamment autour de la Société du Mont Pèlerin ${ }^{2}$, c'est à travers sa forme appliquée se posant en expertise et savoirs au service des gouvernements que le néolibéralisme a pu se diffuser dans le domaine des politiques publiques. C'est dans ce cadre que les think tanks néo-libéraux ont transformé les idées des théoriciens du néo-libéralisme en programme 
d'action. Ces organisations à la frontière entre le champ académique et le champ politique ont été jugées par les historiens du néo-libéralisme comme décisives pour assurer une transformation du discours politique et de l'opinion publique aux États-Unis et au Royaume-Uni ${ }^{3}$.

Les études consacrées aux think tanks en France font souvent état d'une « exception française ${ }^{4}$ » qui rend difficile leur comparaison avec les think tanks tels qu'ils existent aux États-Unis ou au Royaume-Uni. Les limites pour identifier un équivalent des think tanks en France, type d'organisation d'origine américaine ${ }^{5}$, sont à la fois liées à leur faible budget ${ }^{6}$, à leur retrait par rapport aux cabinets ministériels ou aux organismes de réflexion publics qui sont les véritables lieux de décision et d'orientation en matière de politiques publiques ${ }^{7}$, ainsi qu'à leur faible indépendance vis-à-vis de l'État et son administration en raison du financement largement public de la plupart de ces organismes ${ }^{8}$.

Bien que les think tanks en France aient récemment suscité l'intérêt des chercheurs en sciences sociales 9 , peu d'études ont analysé plus spécifiquement les think tanks néo-libéraux et leurs liens avec les réseaux internationaux de promotion $\mathrm{du}$ néo-libéralisme ${ }^{10}$. Pour réaliser ce projet, nous baserons notre étude sur trois organisations françaises ayant diffusé ces idées à un moment clef et qui sont connectées à des réseaux internationaux de promotion de cette pensée : l'Association pour la Liberté Économique et le Progrès Social (ALEPS, fondée en 1966), l'Institut Économique de Paris (fondé en 1982), et Printemps 86 (fondé en 1984). La nature de la première association et son rôle ont déjà fait l'objet de plusieurs travaux.
Parfois considérée comme une Société du Mont Pélerin à l'échelle de la France ${ }^{11}$, ou un think tank patronal ${ }^{12}, l^{\prime}$ 'originalité de son positionnement à ses débuts l'inscrivant dans un néo-libéralisme modéré conservateur à l'allemande a déjà été souligné ${ }^{13}$. La littérature historique rend très peu compte de l'Institut Économique de Paris ${ }^{14}$, certainement en raison de sa faible durée de vie (1982-1987), tandis que Printemps 86 n'a jamais fait l'objet d'une étude approfondie. Nous montrerons que ces trois organisations ont pris des formes imparfaites d'advocacy tanks, c'està-dire celles d'une organisation dont l'objectif est de jouer un rôle dans « la guerre des idées » en tentant d'exercer une influence autant sur l'opinion publique que sur les décideurs politiques ${ }^{15}$.

La thèse de cet article est que l'insertion des militants français dans des réseaux transnationaux néo-libéraux vont les conduire à imiter cette forme particulière d'organisation propice à la diffusion des idées dans le discours politique. Par l'étude de ces cas, nous souhaitons contribuer à la réflexion sur les formes de mobilisation en faveur du néo-libéralisme en mettant en avant l'importance des réseaux internationaux dans le choix des répertoires d'action des militants. Nous soulignons le rôle direct (financement et aide logistique) et indirect (mimétisme) de réseaux internationaux comme la Société du Mont Pèlerin et de l'Institute of Economic Affairs, dans l'institutionnalisation progressive de ces organisations en France. Ils participent ainsi à ce que Lucile Desmoulins appelle une "greffe du modèle d'expertise incarné par les think tanks ${ }^{16}$. » Nous illustrons à partir de plusieurs exemples comment ces think tanks néo-libéraux ont contribué à transformer le discours économique des partis 
politiques de droite français dans le milieu des années 1980.

Pour analyser ce processus de circulation des savoirs et savoir-faire, nous avons dû mettre en place un protocole d'enquête se basant sur des données de natures très différentes. Nous avons consulté les archives d'intellectuels et d'associations internationales néo-libérales (la Société $d u$ Mont Pèlerin ou l'Institute of Economic Affairs) ${ }^{17}$. Ce travail a permis de révéler les liens avec les réseaux intellectuels français en nous basant sur l'analyse des correspondances, des réunions internationales, des comptes rendus d'événements nationaux transmis. Nous avons également réalisé 22 entretiens semi-directifs auprès d'intellectuels français néo-libéraux et responsables d'organisations porteurs de ces idées et témoins de l'époque. Nous avons également consulté leurs mémoires ou leurs autobiographies (le cas échéant). Afin d'évaluer l'audience de ces idées auprès des acteurs politiques et dans le débat public général, nous avons travaillé à partir d'une base de données de presse que nous avons constituée à partir de diverses sources préexistantes : dossiers de presse de la Fondation Nationale des Sciences Politiques, « pressbooks »d'associations, et recherche par mots clefs sur le Periodex (index analytique des périodiques de langue française localisé à la BNF).

\section{L'Association pour la Liberté Économique et le Progrès Social : un « proto-think tank » pour faire connaître les théories néo-libérales}

C'est principalement en fréquentant les réunions de la Société du Mont Pèlerin, ou en rentrant en contact avec des groupes d'universitaires amé- ricains, que les intellectuels néo-libéraux constitués à la fin des années 1970 sous le label des « Nouveaux Économistes » découvrent les idées de l'école de Chicago et de l'école des choix publics ${ }^{18}$. Au-delà de la diffusion académique de ces écrits par des traductions, la rédaction d'ouvrages de vulgarisation, l'organisation de colloques, ils essaient d'établir un type d'organisation nouvelle en France pour faire la promotion de leurs idées.

Les membres français de la Société du Mont Pèlerin ont joué un rôle essentiel pour tenter de mettre en place cette stratégie avec plusieurs tentatives avortées avant l'apogée de leur notoriété dans les années $1980^{19}$. L'inspecteur des finances Jacques Rueff, se faisait, jusqu'à sa mort en 1978, l'intermédiaire entre la première génération des néo-libéraux français qui avaient participé au congrès fondateur de la Société du Mont Pèlerin (Bertrand de Jouvenel, Maurice Allais, Jacques Rueff) et qui incarnait un «néo-libéralisme à la française $\mathrm{e}^{20} »$, et la deuxième génération qui s'est entre-temps américanisé et radicalisé ${ }^{21}$. Il participe à la création de l'Association pour la Liberté Économique et le Progrès Social (ALEPS) en 1966 qui partage certains objectifs des think tanks sans pour autant en partager les ressources ou le format des publications. À des notes concises et ciblées à destination des décideurs publics (on songe aux Hobart Papers de l'Institute of Economic Affairs), l'ALEPS lui préfère l'organisation de réunions publiques et notamment les «Semaines de la pensée libérale» qui se tiennent à la Maison de la Chimie à Paris chaque année pour contrer l'influence du Marxisme. Plus qu'un think tank, il s'agit d'une organisation patronale investie par sa frange conservatrice (Pierre de Calan, hobereau breton en est la figure de proue, 
tandis que le tout est financé par l'Union des Industries et des Métiers de la Métallurgie). Il se pose moins comme un organisme de recherche ou de réflexion que comme un cercle de sociabilité élitiste à la jonction entre l'univers patronal (notamment chrétien), universitaire (économistes membres de la Société du Mont Pèlerin), journalistique (Valeurs Actuelles, La Vie Française) et politique (on retrouve plusieurs Républicains Indépendants à leurs semaines) ${ }^{22}$.

Mais dès cette époque, les néo-libéraux français regardent vers l'étranger pour puiser leurs sources d'inspiration sur la manière de défendre leurs idées. Jacques Rueff conseille à l'économiste Jacques Garello de créer l'équivalent de l'Institute for Economic Affairs ${ }^{23}$, sans donner de suite. Le magazine américain libertarien Reason entretient une correspondance avec les libéraux de l'ALEPS ${ }^{24}$, tandis que tous les membres de cette galaxie effectuent des voyages pouvant s'apparenter à des pèlerinages aux États-Unis et à Londres pour rencontrer les intellectuels et les organisations du mouvement néo-libéral international.

La tendance au renforcement de liens avec l'international se confirme lorsque Jacques Garello, l'un des « nouveaux économistes » membre de la Société du Mont Pèlerin devient président de l'ALEPS en 1980. Il écrit alors : «voici où se situe à mon sens le combat de l'ALEPS pour les années à venir : élargir la brèche libérale, faire pénétrer le souffle de liberté qui nous vient de l'extérieur, [...] il est indispensable de mettre la France à l'heure internationale, dans le domaine des idées comme dans celui de la production et du commerce ${ }^{25}$.»
Ce «souffle de l'extérieur» se matérialise sous la forme de rencontres directes entre des figures internationales du néo-libéralisme et des hommes politiques français à l'initiative de ces médiateurs d'idées. Milton Friedman est invité le 17 octobre 1980 pour s'exprimer au Sénat sur les élections aux États-Unis (en louant les vertus de Ronald Reagan en tant que gouverneur de Californie). L'intellectuel public assure son soutien aux libéraux français en rappelant notamment que le cheminement des idées libérales avait été «chaotique même aux États-Unis, patrie de la libre-entreprise et $d u$ fédéralisme ${ }^{26}$.» Friedrich Hayek est, quant à lui, invité par un groupe de parlementaires de l'UDF (Georges Mesmin, Charles Millon, Alain Madelin, Gérard Longuet, Edmond Alphandéry, Jacques Douffiagues, et François Léotard) et de «nouveaux économistes» (Henri Lepage, Emil Maria Claassen, Jacques Garello, Florin Aftalion, Daniel Pilisi et Pascal Salin) à donner une conférence à l'Assemblée Nationale sur «l'hygiène de la démocratie» à l'occasion de la publication de la traduction de son livre Droit, Législation et Liberté, aux Presses Universitaires de France (Raymond Aron est son discutant $)^{27}$.

\section{« Les idées ont toujours des conséquences » : les think tanks anglais et américains comme modèles de diffusion des idées}

Hormis la Société du Mont Pèlerin qui leur offre une source d'inspiration intellectuelle et des intervenants, une deuxième institution apparaît comme une source d'idées et de méthodes organisationnelles pour les militants néo-libéraux français : 1'Institute of Economic Affairs ${ }^{28}$. Basée à Londres, cette organisation fournit à partir du 
début des années 1980 un soutien intellectuel (par leurs notes et leurs publications), organisationnel (conseils sur le savoir-faire des think tanks) et financier à divers groupes français. L'organisation avait déjà une certaine audience dans les milieux universitaires. Les publications du think tank britannique circulaient alors à l'IEP de Paris par le biais de Jean-Claude Casanova et Jean-Jacques Rosa qui y enseignaient la science économique ${ }^{29}$. Son influence va s'étendre au-delà, auprès de militants en faveur de la libre entreprise.

L'Institut de l'Entreprise (toujours en activité) noue des liens avec l'Institute of Economic Affairs un an après sa création. Quelques-uns de ses membres lui rendent visite en souhaitant s'inspirer de leurs pratiques de diffusion ${ }^{30}$. Antony Fisher, le fondateur de l'organisation britannique se rend plusieurs fois à Paris pour raconter son « histoire à succès », fruit de sa rencontre avec l'intellectuel Friedrich Hayek : «Si vous voulez avoir une influence réelle en politique, le travail essentiel doit être fait de l'extérieur du monde politique; il vous faut d'abord rendre votre plaidoyer intellectuellement acceptable dans le monde intellectuel et principalement dans les milieux universitaires ${ }^{31}$. » De même, l'émissaire américain de la mouvance des think tanks américains en France, l'historien Léonard Liggio, spécialiste du libéralisme français du XIX ${ }^{\mathrm{e}}$ siècle, tient un discours similaire. En tant que représentant d'Atlas Network ${ }^{32}$, il est invité à Paris pour «expliquer la réussite de Ronald Reagan» en mobilisant une formule qui légitime des actions des think tanks en attribuant un rôle performatif aux idées dont il fait la promotion : «ideas have always consequences ${ }^{33}$.»
L'expérience la plus aboutie de création d'un think tank néo-libéral «à la française» construit avec les mêmes objectifs que les organisations anglo-saxonnes est l'Institut Économique de Paris. Il s'agit d'une création des membres les plus militants et insérés dans les réseaux néolibéraux internationaux (notamment le journaliste Henri Lepage et l'économiste Pascal Salin) qui prendra la forme la plus proche d'un think tank produisant des notes à l'égard des décideurs. Il est créé en 1982, directement en réaction à l'élection de François Mitterrand en 1981 qui fait peser de nombreuses craintes sur ces militants néolibéraux par rapport aux orientations économiques de son gouvernement.

Il est fondé par Guy Plunier, un homme issu du monde des affaires (Yves Rocher) qui a été initié aux idées néo-libérales lors de réunions de la Société du Mont Pèlerin ${ }^{34}$. L'Institut Économique de Paris se «donne pour objectif de fournir les instruments intellectuels nécessaires à l'apparition d'une nouvelle culture économique après des décennies de domination presque absolue d'une pensée dirigiste surannée $e^{35}$.» Son comité de patronage lui assure un certain prestige en incluant des Prix Nobel d'économie ou des personnalités à la renommée internationale (Friedrich Hayek en est le président, l'économiste Gordon Tullock est membre du comité de patronage). Dans un document de présentation confidentiel (destiné vraisemblablement aux donateurs), l'intention de s'insérer dans un réseau plus large d'organisations néo-libérales est évident : «les exemples étrangers attestent de l'utilité d'un organisme comme l'Institut Économique de Paris». Outre l'exemple de l'Institute of Economic Affairs, une vingtaine de think tanks 
comme le Manhattan Institute (États-Unis) ou le Fraser Institute (Canada) sont cités.

Antony Fisher, directeur de l'Institute of Economic Affairs, contacte lui-même des chefs d'entreprise britanniques pour assurer le financement de l'Institut qu'il vient inaugurer en personne à Paris à la fin de l'année 1982, tandis que le délégué de l'Institut, Guy Plunier se rend à Londres à plusieurs reprises pour comprendre les conditions de la réussite de l'Institute of Economic Affairs en Angleterre afin de la reproduire en France $^{36}$. L'entreprise intellectuelle est soutenue par la mairie de Paris qui verse 385000 francs de subventions sur la période, ainsi que l'organisation internationale Atlas Network ${ }^{37}$ mise en place par Antony Fisher en 1981 pour encourager la création et le développement de think tanks libéraux et conservateurs dans le monde ${ }^{38}$.

Certains «nouveaux économistes » néo-libéraux gravitant autour de l'Institut Économique de Paris contribuent aux publications de 1'Institut britannique en écrivant sur la situation qu'il considère inquiétante en France ou en dressant le portrait de célèbres figures libérales comme l'économiste français Frédéric Bastiat ${ }^{39}$. Les britanniques viennent quant à eux présenter les vertus de la révolution thatchérienne à Paris devant des groupes proches de l'opposition. Fort de ces soutiens, l'Institut Économique de Paris a produit, sur la période 1982-1987, une douzaine de petits livres en lien avec les politiques publiques ou la pensée économique néo-libérale ${ }^{40}$, organisé 96 petits déjeuners et conférences et 10 colloques. Ce sont ces formes de « mobilisations silencieuses » qui permettent de diffuser la bonne parole dans certains milieux de l'opposition à une époque où les clubs politiques foisonnent pour renouveler le discours de la droite ${ }^{41}$. L'Institut investit particulièrement la question des zones franches auxquelles il consacre plusieurs colloques qui alimentent la réflexion du futur ministre de l'Industrie Alain Madelin. Il mettra lui-même en place certaines expérimentations en la matière dans sa circonscription de Bretagne ${ }^{42}$.

Les petits déjeuners qu'organise de manière assez confidentielle l'Institut voient défiler tout ce que la France compte d'auteurs néo-libéraux qui présentent leurs livres avec des thèmes clefs de l'opposition, «Désétatiser» (Bertrand Jacquillat), «Individualisme» (Alain Laurent), «La liberté à refaire» (Bertrand Lemennicier), «Les fondements du libéralisme» (Jean-Marie Benoist $)^{43}$. Des figures néo-libérales internationales viennent raconter leurs pays, notamment Madsen Pirie, président de l'Adam Smith Institute qui vient faire une présentation sur les «Privatisations en Grande-Bretagne» en décembre 1984. L'Institut Économique de Paris, comme le Club de l'Horloge à l'époque dans sa version conservatrice $^{44}$, pose les jalons d'une sociabilité militante transpartisane : sont invités aussi bien le président du Centre National des Indépendants et Paysans (Philippe Malaud), le directeur des études du Front National (Jean-Yves le Gallou), qu'un délégué du RPR ou un député UDF.

Le rôle de l'Institut Économique de Paris est également de diffuser les livres libéraux étrangers en France. Pour ce faire, une librairie est créée en 1985 et propose un catalogue extrêmement complet comprenant les notes de l'Institute of Economic Affairs ${ }^{45}$. L'Institut Économique de Paris est extrêmement proche du $\mathrm{n}^{\circ} 2$ du Parti 
Républicain (UDF), Alain Madelin, qui est l'un des principaux clients de la maison d'édition ${ }^{46}$ et dont il fait la promotion de manière indirecte en citant les ouvrages dans la chronique qu'il écrit à l'époque dans Le Figaro Magazine intitulée «Pour faire aimer le libéralisme ${ }^{47}$.» Il préside le groupe de recherche et d'études sur les privatisations émanant de l'Institut Économique de Paris qui organise plusieurs colloques à Paris en présence de conseillers de Margaret Thatcher ${ }^{48}$.

L'importance de l'Institut Économique de Paris est cependant à nuancer au regard de sa faible audience médiatique et du fait de l'interruption précoce de ses activités liées à des querelles personnelles en $1987^{49}$. Elle témoigne cependant d'une volonté d'imitation d'expériences étrangères et surtout l'existence d'un soutien organisationnel et financier au niveau international.

\section{Une harmonie entre théoriciens et praticiens du libéralisme pour penser le programme économique des droites}

Le contexte politique précédant les élections législatives de 1986 nous offre un bon aperçu de la capacité de ces jeunes think tanks français de faire entendre leur philosophie et ses traductions concrètes dans le domaine des politiques publiques auprès des organisations partisanes de droite.

Le congrès régional de la Société du Mont Pèlerin organisé le $1^{\text {er }}$ mars 1984 à Paris par l'Institut Économique de Paris témoigne de la synergie entre les intellectuels néo-libéraux français, internationaux, et une partie des droites françaises implantées dans les médias et dans les organisa- tions partisanes. Le congrès bénéficie du concours financier d'Edwin Feulner qui est alors président de l'Heritage Foundation (think tank libéralconservateur américain) et trésorier de la Société $d u$ Mont Pèlerin ${ }^{50}$, celui de grandes entreprises françaises comme Nina Ricci, Michelin ou Yves Rocher, celui du groupe de presse L'Express, et celui de la Mairie de Paris ${ }^{51}$. La liste des invités offre un aperçu de différents réseaux de promotion du néo-libéralisme à droite : des «Nouveaux économistes» (Jacques Garello, Pascal Salin, Henri Lepage, Emil Maria Claassen, Florin Aftalion), des représentants du patronat (François Ceyrac du CNPF, Michel Drancourt, Serge Dassault, Jacques Riboud), des journalistes français (Valeurs Actuelles, La Vie Française, L'Express et Le Figaro), des journalistes internationaux (Wall Street Journal, Newsweek etc.) et des acteurs politiques de droite comme Yvan Blot et Alain Juppé, tous les deux au RPR.

Le programme est ponctué de rencontres, comme cette réception organisée au siège du magazine L'Express ou la présentation de papiers sur l'«ordre économique mondial». Le point d'orgue de l'évènement est la cérémonie de remise de la médaille de Vermeil de Paris à Friedrich Hayek par le maire Jacques Chirac. L'initiative de cette rencontre provient de la Mairie de Paris par l'entremise de l'économiste Pascal Salin. Ce dernier s'en réjouit auprès de Friedrich Hayek : «Je considère significatif le fait qu'il ait voulu vous voir. Mon impression est que Jacques Chirac est de plus en plus influencé par de bonnes idées libérales ${ }^{52}$.»

Le Maire de Paris prononce un vibrant discours à l'Hôtel de Ville de Paris - écrit par le «Nouveau 
philosophe» Jean-Marie Benoist ${ }^{53}$ - en hommage à l'œuvre du Prix Nobel d'économie qui a alors 85 ans. Il reprend à son compte ses principales idées comme la critique du concept de «justice sociale» ou de l'interventionnisme : «il devient alors manifeste que l'interventionnisme débouche sur l'État bureaucratique qui se substitue aux individus pour déterminer eux-mêmes leurs besoins et leurs préférences ${ }^{54}$.» Chirac loue alors le philosophe qui a analysé les ressorts du socialisme dépeint en régression intellectuelle vers le «tribalisme» et des modes de pensée archaïques qui entraînent vers la «Route de la servitude». Il cite sa «Constitution de la Liberté» et sa définition de la loi d'un point de vue libéral. Il (ou plutôt sa plume) la rapproche de la limitation du pouvoir selon le philosophe français Alain.

Le discours, même s'il est largement déterminé par son contexte d'élocution, témoigne en tout cas de la volonté des droites de s'appuyer sur des références intellectuelles internationales légitimant leur discours et leurs futures actions. Friedrich Hayek dans son discours de clôture du Congrès (traduit et publié dans Le Figaro Magazine) peut se féliciter quant à lui du chemin parcouru par la Société du Mont Pèlerin et rend hommage à la nouvelle génération des «Nouveaux Économistes» en France. Il conclut en faisant la synthèse de ses conceptions économiques et philosophiques qui entrent en résonance avec le libéralisme conservateur des réseaux français qui l'accueillent à Paris : «Nous devons retourner à un monde où notre vie est guidée non pas par la seule raison, mais par la raison et la morale, en partenaires égaux, où la vérité de la morale est tout simplement celle d'une tradition morale, celle de l'Occident chrétien qui a façonné la morale de la civilisation modern ${ }^{55}$.» Ce congrès est considéré par les membres de la Société $d u$ Mont Pèlerin comme un hommage aux libéraux classiques et témoigne du regain de ces idées au point d'être considéré comme "l'un des meetings régionaux les plus réussis de l'histoire de la Société56.»

En France, la couverture médiatique assez large du passage dans la capitale du penseur d'origine viennoise témoigne du succès des médiateurs du néo-libéralisme à mettre leurs thèmes au cœur du débat intellectuel et politique au milieu des années 1980. Les journaux de droite font un accueil très favorable au fondateur de la Société du Mont Pèlerin : Le Figaro Magazine fait de Paris la " capitale du libéralisme », et constate le développement de clubs et d'associations qui relaient le message politique du libéralisme hayékien dans les partis ${ }^{57}$. La gauche couvre ce qui est perçu comme un retour du libéralisme, mais de manière critique : Le Monde réalise un entretien avec Friedrich Hayek, jugé «réactionnaire» et «gourou du libéralisme ultra ${ }^{58}$ », tandis que le Nouvel Observateur par la plume de Jacques Julliard consacre un long papier à la «nouvelle idole de la droite». Il y restitue le contenu de son œuvre maîtresse Droit, Législation et Liberté et voit dans le succès de Hayek un prélude pour une domination durable des idées des droites en France. Pour lutter contre ses idées, l'éditorialiste propose des pistes de réflexion à la gauche sous forme de concessions révélatrices du climat intellectuel de l'époque. Il propose notamment d'en finir avec le fantasme de la table rase et à «réconcilier le socialisme avec l'esprit d'entreprise ${ }^{59}$.» 
La stratégie visant à faire entendre la pensée des intellectuels libéraux auprès des décideurs de tous les partis se poursuit avec l'organisation d'une «croisière libérale» en Méditerranée intitulée «Cap sur le libéralisme» en novembre 1985 à l'initiative de sympathisants du Parti Républicain avec l'ensemble des clubs et organisations néo-libérales de l'époque qui se mobilisent pour avoir l'oreille de l'opposition. Hommes politiques ou conseillers (on retrouve Yvan Blot, conseiller au RPR et Henry de Lesquen), intellectuels (des Nouveaux Économistes, les philosophes libéraux Philippe Nemo et Alain Laurent), chefs d'entreprise (dont Michel-Édouard Leclerc), se retrouvent sur le même bateau pour réfléchir au programme de l' «après-socialisme ${ }^{60}$.» Au cours de cette croisière de quatre jours, les conférences s'enchaînent pour discuter de tous les domaines de politiques publiques dans une perspective libérale radicale destinée à convaincre les plus modérés du bateau (principalement des acteurs politiques) : dénationalisation de la monnaie (Philippe Nataf), politique répressive en matière judiciaire (Xavier Raufer), abolition du contrôle des loyers (Georges Mesmin), école à la carte, chèque éducation de Friedman, privatisation des universités (Roland Drago, Alain Madelin), déréglementation des entreprises (HenriLepage), réduction du nombre de fonctionnaires (Yvan Blot), remise en question de l'existence du service public (Bertrand Jacquillat), privatisation de certains pans de la sécurité sociale (René de Laportalière, Jacques Garello), rendre le pouvoir au consommateur (Alain Madelin, Michel-Édouard Leclerc) ${ }^{61}$. Toutes ces mesures radicales n'ont pas été prises en compte dans le programme des droites ou dans le discours public. Les journalistes présents à bord n'ont d'ailleurs pas manqué de noter l'opposition entre les théoriciens universitaires et les praticiens à bord ${ }^{62}$. Cependant, ces propositions et la diffusion d'un tel discours contribuent à nourrir la réflexion des droites dans la préparation des élections législatives de 1985.

C'est à partir de cette volonté de constituer un programme commun des droites sur cette base, en mobilisant le répertoire d'action des think tanks américains, que René de Laportalière fonde Printemps 86 en 1984. Diplômé de l'Institut d'Études Politiques de Paris, ancien militaire, il est devenu cadre de banque et s'est investi au RPR en créant «RPR banques» peu après la victoire de François Mitterrand à l'élection présidentielle de 1981, afin de lutter contre la politique du crédit du gouvernement. Il quitte le RPR suite à des désaccords avec des conseillers de Jacques Chirac et décide de réunir des fonds privés et une vaste équipe pour préparer des textes de loi « clefs en main » en dehors des partis pour le futur gouvernement de droite. Là aussi, l'influence américaine est palpable : c'est la lecture du livre de Milton Friedman, $L a$ tyrannie du statu quo ${ }^{63}$, qui l'incite à créer cette structure transpartisane. Le lauréat du prix Nobel d'économie américain considérait que pour qu'un programme libéral soit efficace, il devait être soigneusement préparé en avance pour être appliqué dans les six premiers mois après l'élection du président afin de prévenir l'action des groupes de pression pour bloquer les réformes ${ }^{64}$. René de Laportalière s'entoure d'économistes et de hauts fonctionnaires pour rédiger les textes de loi enregistrés précieusement sur disquette et envoyés à des listes de diffusion 
sur Minitel. Il s'appuie sur un conseil politique composé de membres de l'opposition les plus libéraux au sein de toutes les tendances (Alain Madelin pour le PR, Jacques Toubon pour le RPR, et Charles Million pour les barristes $)^{65}$.

Ses convictions libertariennes l'amènent à penser que la victoire des droites n'est pas une condition suffisante pour redonner du pouvoir à la société civile. Cette dernière devait pour cela se reprendre en main et générer elle-même des propositions précises et éviter de dépendre de la bonne volonté d'hommes vivant de l'argent de l'État pour les appliquer ${ }^{66}$. Il profite de son amitié avec James Goldsmith, le détenteur du magazine L'Express pour diffuser ses idées auprès de ce lectorat. Le programme qu'il prépare avec ses experts est livré à l'été 1985 et relayé par les journaux de l'opposition. Bien que radicales (ouverture à la concurrence des caisses d'assurance maladie ou suspension de l'application du SMIC, fin du financement public des syndicats), elles sont néanmoins entendues par des acteurs politiques qui participent aux ateliers préparatoires comme le futur secrétaire d'État Gérard Longuet (Parti Républicain, UDF) ${ }^{67}$.

Cette initiative constitue précisément une tentative d'imposer dans la vie politique française une nouvelle forme de militantisme correspondant à celle des advocacy tanks, une catégorie de think tanks dont l'objectif principal est de défendre une idéologie politique en développant une expertise sur les politiques et en émettant des recommandations précises ${ }^{68}$. René de Laportalière privilégie ce modèle d'action indépendamment des partis et recourt à l'expertise technique afin de faire triompher ses idées de manière plus efficace que des clubs politiques et émanciper le triomphe du libéralisme de personnalités politiques particulières.

L'esprit général des propositions émises par printemps 86 ou lors de la «croisière libérale» sera en grande partie retenu par les rédacteurs du programme commun des droites ${ }^{69}$. Ils en tempéreront néanmoins quelque peu la radicalité pour en faire un programme politique capable d'être accepté par toutes les composantes de l'opposition et l'opinion publique française qui est alors loin d'être acquise au néo-libéralisme ${ }^{70}$.

\section{L'héritage de la première génération de clubs et la fragmentation du mouvement néo- libéral}

Après les élections législatives de 1988 et un passage au pouvoir des droites soldé par de fortes divisions et une mise à l'écart des plus fervents partisans du néo-libéralisme (« la bande à Léo »), les clubs politiques et think tanks néo-libéraux connaissent une certaine traversée du désert illustrée par la mise en sommeil de nombreuses organisations (Printemps 86 et l'Institut Économique de Paris mettent fin à leurs activités). Cependant, la première génération de clubs et d'associations politiques a formé une plus jeune génération de militants néo-libéraux qui a participé à une vague de fondation de think tanks à l'américaine au début des années $2000^{71}$.

L'un des premier (qui se développe réellement au cours des années 2000), la Fondation iFRAP, est fondée en 1986 par Bernard Zimmern. Il faisait alors partie de la mouvance néo-libérale (il participait aux réunions de l'ALEPS) et a eu 
l'idée de fonder son think tank directement sur le modèle du think tank américain conservateur Heritage Foundation $^{72}$. De même, on peut faire remonter la création de l'Institut Montaigne en 2000 à des initiatives similaires élaborées au sein des réseaux néo-libéraux des années 1980. L'Institut La Boétie fondé en 1978 par Bernard de La Rochefoucault est son ancêtre ${ }^{73}$. Celui-ci avait participé à la «croisière libérale» de 1985 et avait été mis en relation avec l'Institute of Economic Affairs par Guy Plunier ${ }^{74}$. Ses rencontres avec le think tank britannique avaient nourri le modèle d'organisation qu'il voulait alors créer.

Le néo-libéralisme a connu un certain renouveau dans sa diffusion par l'émergence de cette vague de think tanks au début des années 2000 liée à l'arrivée d'une nouvelle génération militante et le développement d'internet qui a permis de baisser le coût de coordination des militants néo-libéraux français. L'encadré 1 présente une liste des principales organisation néo-libérales au début des années 2010.

\section{Encadré 1}

Principales organisation néo-libérales en activité

\section{Fondation iFRAP (dirigée par Agnès Verdier-Molinié depuis 2009) \\ La Fondation pour la Recherche sur les Administrations et les Politiques publiques a été fondée en 1985 par le chef d'entreprise Bernard Zimmern. Elle est aujourd'hui dirigée par la très médiatique Agnès Verdier-Molinié et se focalise principalement sur l'administration publique française et les gaspillages d'argent public. À l'instar des think tanks américains, son équipe importante d'experts diffuse régulièrement ses propositions concrètes par le}

biais de ses notes, ses études et son journal Société Civile. On retrouve dans les propositions de l'iFRAP pour 2012 des idées comme la suppression du SMIC au profit d'un salaire minimum négocié dans chaque entreprise, la généralisation de l'ouverture des données publiques, l'augmentation du temps de travail des enseignants...

\section{Institut économique Molinari (2003)}

Il est dirigé par Cécile Philippe, ancienne étudiante de Pascal Salin (Président de la Société du Mont Pèlerin de 1994 à 1996), titulaire d'un doctorat en science économique préparé en partie au Mises Institute à Auburn. Du nom de l'un des précurseurs de l'anarchocapitalisme, l'Institut vise, par sa présence dans les médias et par ses notes, à offrir une analyse de l'actualité économique inspirée de l'école autrichienne. II s'est fait connaître en participant à l'élaboration de le « Journée de libération fiscale » (inspirée des think tanks américains qui rapportent les prélèvements obligatoires sur la durée d'une année pour déterminer ce jour). Cet institut a consacré ses travaux à une critique du GIEC et des politiques publiques luttant contre le réchauffement climatique où l'inefficacité de l'euro (par rapport à une situation de concurrence monétaire). Sa directrice Cécile Philippe est souvent présente sur les plateaux de télévision (notamment BFM) ou les radios pour commenter l'actualité.

\section{Institut Montaigne (2000)}

Cette organisation a été créée en 2000 par Claude Bébéar, Président d'Axa et est devenu en l'espace d'une décennie le think tank doté du budget le plus important (environ 3 millions d'euros en 2013). Généraliste et d'orientation libérale modérée, l'Institut Montaigne a pour objectif de produire des études et des rapports de grande ampleur analysant la société française et ses problèmes afin de proposer des solutions clefs en main pour les décideurs politiques. L'Institut a par 
exemple commandé une étude de grande ampleur au chercheur Gilles Kepel pour dresser un état des lieux des banlieues en France qui a donné lieu à la publication d'un livre. À côté de ce type de publications, I'Institut Montaigne produit également des rapports comprenant des mesures concrètes sur un thème précis. C'est le cas par exemple d'un rapport publié en décembre 2013 proposant des mesures chiffrées pour réduire le déficit public : l'allongement de la durée de travail des fonctionnaires ou la réduction du nombre de dispositifs d'aide aux entreprises...

\section{La Fondation pour I'Innovation Politique (2004)}

La Fondation pour I'Innovation Politique (Fondapol) a été créée en 2004 par Jérôme Monod, conseiller de Jacques Chirac et avait pour vocation d'être un think tank au service de l'UMP se définissant comme rattaché aux « idées de la droite et du centre ». II était principalement destiné à rapprocher les intellectuels de l'UMP (le philosophe Bruckner et le sociologue Raymond Boudon participent aux premières réflexions du think tank) et financé par les fonds de Matignon à sa création. Dirigé depuis 2008 par Dominique Reynié, professeur à Sciences Po (et auteur d'un mémoire de DEA sur la pensée de Friedrich Hayek), il n'a plus de lien structurel avec I'UMP et reçoit des subventions publiques en sa qualité de Fondation reconnue d'utilité publique. D'orientation intellectuelle libérale et en faveur de la construction européenne, cette Fondation organise ses activités de réflexion à partir de contributeurs issus du monde universitaire qui rédigent des notes sur différents sujets économiques, politiques et sociaux. Elle diffuse ses notes aux décideurs et journalistes, mais tente de s'adresser à un public plus large à l'aide de l'organisation de débats, conférences publiques et grâce à son blog "Trop Libre.fr ». La Fondapol a joué à plusieurs reprises un rôle de propositions, notamment au cours de l'élection présidentielle de
2012. L'idée de règle d'or budgétaire consistant à inscrire dans la constitution l'interdiction de voter des budgets en déficit proposé dans une note a été reprise par plusieurs personnalités politiques. Elle accueille des contributions de natures assez diverses allant de celles de Pascal Salin sur la monnaie à celles de plus jeunes universitaires qui ne sont pas nécessairement d'obédience libérale.

\section{GénérationLibre (2013)}

Génération Libre a été fondé par le philosophe Gaspard Koenig (normalien et ancienne plume de la ministre Christine Lagarde) en 2013. Sa première originalité est de militer à la fois pour la libéralisation de l'économie française (libéralisme économique) et les libertés individuelles (libéralisme sociétal). Sa seconde originalité est de mobiliser une rhétorique générationnelle dans son argumentation, notamment en mettant en avant l'idée que l'État-providence a profité aux générations précédentes au détriment de celle du fondateur du think tank (trentenaire). En quelques années d'existence GénérationLibre est à l'origine d'un grand nombre d'études et de très nombreux passages médiatiques. Son fondateur, Gaspard Koenig, est chroniqueur régulier dans $L e$ Point, et Les Échos. Le think tank a produit une étude visant à remettre au cœur du débat l'idée de revenu universel (déjà imaginé sous forme d'impôt négatif par Milton Friedman). L'idée est de mettre en place d'un côté un impôt proportionnel unique (Libertaxe) sur les revenus qui viendrait se substituer à l'IR, la CSG, et à l'impôt sur les sociétés, et de l'autre un revenu universel (LIBER) se substituant à toutes les différentes allocations conditionnelles.

\section{L'Institut de Recherche Économique et Fiscale (2002)}

Fondé en 2002 par des acteurs issus de l'entreprise, il est dirigé aujourd'hui par Nicolas Lecaussin, diplômé 
de Sciences Po Paris et ancien membre des cercles Idées-Action d'Alain Madelin, et de l'iFRAP. Ce think tank produit des études de politiques publiques courtes à destination des journalistes portant essentiellement sur les politiques économiques et fiscales. Avec son président Jean-Philippe Delsol, ils sont souvent invités dans les médias comme Atlantico et l'Opinion pour commenter l'actualité. Ils sont auteurs du livre À quoi servent les riches ? (2012). Son conseil scientifique comprend des économistes néo-libéraux de la Société du Mont Pèlerin.

Sources : sites internet de ces organisations.

Cette liste témoigne de certains liens avec les clubs libéraux des années 1980 les plus internationalisés, mais aussi d'un alignement croissant sur le modèle des think tanks à l'américaine qui recourent à l'expertise d'universitaires pour organiser des événements et produire du contenu à destination des décideurs. Cela permet d'observer une certaine convergence vers le modèle de l'advocacy tank, visant à mettre en avant des propositions de politiques publiques concrètes, alors que la première génération de think tanks néo-libérausx français offrait un contenu plus académique et visant à la promotion de l'œuvre des figures du néo-libéralisme comme Friedrich Hayek.

On observe aussi une certaine professionnalisation du mouvement qui se manifeste par le développement de capacités financières et en ressources humaines qui permettent à ces think tanks "nouvelle génération" d'être des voix indépendantes des partis ou des universités. Elles sont dotées chacune de quelques salariés et de budgets pouvant aller jusqu'à quelques millions d'euros (comme la Fondation pour l'Innovation Politique). Autre fait notable, l'influence du modèle de think tank à l'américaine est encore plus présent pour les think tankers de cette nouvelle génération. Philippe Manière, directeur de l'Institut Montaigne de 2004 à 2009 dit s'être inspiré de voyage aux États-Unis et de son modèle de think tank, Cécile Philippe (Institut Économique Molinari) a réalisé une partie de sa thèse dans un think tank américain, Agnès Verdier-Molinié a participé à la Bourse Tocqueville dont l'objectif est d'envoyer des jeunes Français découvrir les méthodes des think tanks américains, tandis que le fondateur de l'iFRAP lui-même avait été marqué lors de son séjour aux États-Unis par Heritage Foundation $^{75}$.

Cette nouvelle génération de néo-libéraux mobilise davantage le registre de l'expertise plutôt que celui de l'idéologie. La manière dont Agnès Verdier-Moliné présente son travail incarne bien cette volonté de se mobiliser de manière plus silencieuse, objectivante et moins partisane en faveur de l'idéologie néo-libérale : « " Dire qu'il faut baisser la dépense publique et les impôts, ce n'est pas être libéral, c'est être objectif [...]. L'étiquette, ce n'est pas moi qui l'ai choisie et ça m'est égal. L'idée est de faire nos études sérieusement, de mettre nos données sur la place publique. Elles peuvent servir à un gouvernement de gauche comme de droite. D'ailleurs, le clivage n'est pas clair, il y a des gens de droite étatistes ${ }^{76}$. »

Une sociologie récente du mouvement libertarien en France montre comment Internet favorise une nouvelle forme de militantisme. L'accès aux œuvres philosophiques néo-libérales est plus 
aisé, tout comme l'accès aux autres militants internationaux grâce aux réseaux sociaux ${ }^{77}$. La structuration de la webosphère montre une certaine émancipation vis-à-vis des organisations de la génération précédente et la promotion d'idées plus libertariennes et moins liées au conservatisme. Par ailleurs, plusieurs organisations passent par des voies extra-académiques pour faire connaître la pensée néo-libérale comme le webzine Contrepoints fondé en 2009 qui fait converger des billets d'actualité écrits par des organisations néo-libérales, l'Institut Coppet qui met en libre accès des centaines de textes d'auteurs libéraux français du XVIII ${ }^{e}$ et XIX ${ }^{e}$ siècle, ou encore le groupe d'activistes Students for Liberty ${ }^{78}$.

Il existe désormais un groupe relativement important de jeunes intellectuels et experts néo-libéraux qui sont présents dans les médias pour porter sous un autre registre les idées défendues par la génération des Nouveaux Économistes (voir l'entretien avec Gaspard Koenig dans ce numéro) ${ }^{79}$. La nouvelle vague de création de think tanks est allée de pair avec une médiatisation accrue de militants néo-libéraux.

$* * *$

Notre étude historique a mis au jour les transformations des principaux acteurs et canaux de diffusion de la pensée néo-libérale en France avec la greffe du modèle des think tanks au début des années 1980. L'ALEPS, l'Institut Économique de Paris et Printemps 86 se sont peu à peu imposés en vulgarisateurs des thèses néo-libérales avec l'aide d'organisations britanniques et américaines. Ces organisations se sont posées en intermédiaires entre les intellectuels et les organisations internationales néo-libérales et le contexte politique français. Elles se sont progressivement institutionnalisées en think tanks dans la mesure où ces organisations sont devenues une "passerelle entre ceux qui ont des idées et rêvent d'avoir le pouvoir, et ceux qui ont le pouvoir et veulent appuyer leur action sur une analyse réfléchie $»^{80}$. Elles se sont transformées en advocacy tanks en incarnant deux fonctions : un rôle de recherche sur des questions de politiques publiques et un rôle encore plus flagrant de connecteurs de réseaux et d'échange d'idées auprès d'acteurs politiques avec lesquels ils avaient tissé préalablement des liens personnels. Ces formes de mobilisations leur ont permis de contribuer à faire évoluer le discours économique français des droites à la veille des élections législatives de 1986. Les tentatives d'importation du modèle des think tanks en France nous semblent témoigner de la volonté de certaines associations et groupements intellectuels français d'imiter ces organisations afin d'exercer un pouvoir politique à partir d'une légitimation de savoirs issus de sciences sociales qui viendraient concurrencer ceux de l'expertise d'État.

Pour reprendre la distinction des rôles politiques de l'intellectuel proposés par Lipset et Basu ${ }^{81}$, le néo-libéralisme a connu ses prêtres mobilisant leur intellect pour définir les contours d'une utopie néo-libérale dans sa version la plus radicale au cours des années 1970-1980. Mais, à cette époque, elle n'avait pas ses experts mobilisant leur intelligence pour élaborer une stratégie incrémentale visant à transformer leur idéologie en une ingénierie technique de nature à influencer le discours des acteurs partisans et l'orientation 
des politiques publiques. Depuis les années 2000, le discours et la mobilisation des néo-libéraux français s'apparente à cette forme d'engagement intellectuel.

Il est encore trop tôt pour évaluer avec suffisamment de recul si l'impact des think tanks néolibéraux les plus récents sur le système politique français. Il est difficile d'objectiver leur influence et leur capacité à infléchir les décisions en matière de politique publique dans un contexte où la « structure des régimes des savoirs » est encore dominée, en France, par l'expertise des ministères et des organismes de recherche liés au gouvernement ${ }^{82}$. Le classement de l'influence des think tanks réalisé régulièrement par James Mc Gann, malgré ses limites méthodologiques, est là pour nous le rappeler : très peu de think tanks français apparaissent dans le classement et ceux qui y font une apparition sont souvent dépendants de l'État, alors que l'indépendance est considérée comme un des éléments caractérisant les think tanks ${ }^{83}$.
$\mathrm{N} \cdot \mathrm{O} \cdot \mathrm{T} \cdot \mathrm{E} \cdot \mathrm{S}$

1. Friedrich August von Hayek, « Les intellectuels et le socialisme ", Commentaire, vol. 99, $\mathrm{n}^{\circ} 3$, 2002, p. 674-675.

2. Organisation élitiste comprenant les figures intellectuelles internationales du néo-libéralisme au niveau international et fondée en 1947 par l'économiste autrichien Fredrich Hayek (prix Nobel 1974). Philip Mirowski et Dieter Plehwe (dir.), The Road from Mont Pèlerin: The Making of the Neoliberal Thought Collective, Cambridge, Harvard University Press, 2009 ; Angus Burgin, The great persuasion: reinventing free markets since the Depression, Harvard University Press, 2012.

3. Richard Cockett, Thinking the Unthinkable: ThinkTanks and the Economic Counter-Revolution 19311983, London, Harper Collins, 1995 ; Thomas Medvetz, « Les think tanks aux États-Unis. L'émergence d'un sous-espace de production des savoirs ", Actes de la recherche en sciences sociales, vol. 176-177, $\mathrm{n}^{\circ} 1$, 2009, p. 82-93 ; Richard Heffernan, "'Blueprint for a revolution'? The politics of the Adam Smith institute", Contemporary British History, vol. 10, $\mathrm{n}^{\circ}$ 1, 1996, $\mathrm{p}$. 73-87.

4. Lucile Desmoulin, "French Public Policy Research Institutes and Their Political Impact as Another Illustration of the French Exception", in James G. McGann et Richard Kent Weaver (dir.), Think Tanks \& Civil Societies: Catalysts for Ideas and Action, New Brunswick, Transaction Publishers, 2000, p. 139-168; Catherine Fieschi et John Gaffney, "French Think Tanks in a Comparative Perspective", in Diane Stone, Andrew Denham et Mark Garnett (dir.), Think Tanks Across Nations : a Comparative Approach, Manchester, Manchester University Press, 1998, p. 42-58.

5. Anne Deysine, « Les Think-Tanks : spécificité 
américaine ou produit d'exportation ? », Le Banquet, $\mathrm{n}^{\circ}$ 30, 2012, p. 113-131; Evelyne Joslain, L'Amérique des think tanks : un siècle d'expertise privée au service d'une nation, Paris; Budapest, L'Harmattan, 2006.

6. Patrick Papazian, « Think tanks...à la française! Évolution et moyens des groupes de réflexion », in Guillaume Bernard et Éric Duquesnoy (dir.), Rapport Anteios. Les forces politiques françaises: genèse, environnement, recomposition, Presses universitaires de France, 2007, p. 181-185; Kevin Brookes et Benjamin Le Pendeven, « L'État innovant (1) : renforcer les think tanks », in Innovation politique 2014, Presses Universitaires de France, 2014, p. 387-425.

7. John Gaffney, "The Political Think-Tanks in the UK and the Ministerial Cabinets in France", West European Politics, vol. 14, no 1, 1991, p. 1-17 ; John L. Campbell et Ove K. Pedersen, The National Origins of Policy Ideas: Knowledge Regimes in the United States, France, Germany, and Denmark, Princeton University Press, 2014.

8. Stephen Boucher et Martine Royo, Les think tanks : cerveaux de la guerre des idées, Paris, Félin, 2006, p. 78 .

9. Lucile Desmoulins, Le rôle des think tanks dans les processus de décision de politique extérieure: analyse comparée, Lille, France, Atelier national de Reproduction des Thèses, 2006 ; Marc Patard, La démocratie entre expertise et influence: le cas des think tanks français (1979-2012), IEP de Paris, Thèse pour l'obtention du doctorat de science politique, 2012 ; Geoffrey Geuens (dir.), « Think tanks, experts et pouvoirs », Quaderni, no 70, 2009.

10. Notons le travail de thèse de François Denord sur les réseaux néo-libéraux français des années 1930 aux années 1950 (dont les idées sont éloignées du néolibéralisme contemporain que nous étudions dans cet article). François Denord, Néo-libéralisme, version française : histoire d'une idéologie politique, Paris,
Demopolis, 2007.

11. Serge Audier, Néo-libéralisme(s) : une archéologie intellectuelle, Paris, Grasset, 2012, p. 160 ; François Denord, « Le prophète, le pèlerin et le missionnaire : la circulation internationale du néo-libéralisme et ses acteurs ", Actes de la recherche en sciences sociales, vol. $145, \mathrm{n}^{\circ} 5,2002$, p. 15.

12. Michel Offerlé, Sociologie des organisations patronales, Paris, France, la Découverte, 2009, p. 42. 13. Michael C. Behrent, "Justifying Capitalism in an Age of Uncertainty: L'Association pour la Liberté Économique et le Progrès Social, 1969-1973", in Émile Chabal (dir.), France since the 1970s History, Politics and Memory in an Age of Uncertainty, Bloomsbury Academic, 2014 ; Kevin Brookes, Diffusion et transformation du néo-libéralisme en France des années 1960 aux années 1980 L'Association pour la Liberté et le Progrès Social et les "Nouveaux Économistes 》, mémoire de master 2 sous la direction d'Emmanuel Taïeb, Institut d'Études politiques de Grenoble, Grenoble, 2012.

14. À l'exception de François Denord qui l'évoque brièvement. Cf. François Denord, Néo-libéralisme, version française, op. cit., p. 296.

15. «[Les advocacy tanks] bien qu'ils conservent une indépendance formelle, sont liés à des groupements ou des intérêts idéologiques particuliers. Ils ont tendance à voir leur rôle dans le processus de prise de décision politique comme une guerre des idées à remporter plutôt que comme une recherche désintéressée pour les meilleurs politiques publiques », traduction libre de James G McGann et Richard K. Weaver (dir.), op.cit., p. 7.

16. Lucile Desmoulins, «Profits symboliques et identité (s) : les think tanks entre affirmation et dénégation », Quaderni, n 70, Automne 2009, p. 11-27.

17. Ces archives sont situées à la Hoover Institution sur le campus de l'Université de Stanford. Nous avons 
consulté les fonds suivants : «F.A Hayek Papers »; « Institute of Economic Affairs »; " Mont Pèlerin Society Records ».

18. Kevin Brookes, " Deux réseaux de promotion du néolibéralisme entremêlés dans les années 1960 et 1970 : L'ALEPS et le groupe des "nouveaux économistes" ", in Olivier Dard, Dominique Barjot, Frédéric Fogacci et Jérôme Grondeux (dir.), Histoire de l'Europe Libérale. Libéraux et libéralisme en Europe, $X V I I I^{e}-X X I^{e}$ siècle, Paris, Nouveau Monde Éditions, 2016, p. 245-264.

19. François Denord, Néo-libéralisme, version française, op. cit.

20. Arnaud Diemer, «Le néolibéralisme français ou comment penser le libéralisme au prisme des institutions », Économie et Institutions, n $20-21,2014$, p. 81-113.

21. Kevin Brookes, «Un « libéralisme scientifique » contre les gauches. La réception du néo-libéralisme américain en France dans les années 1970 », Raisons politiques, $\mathrm{n}^{\circ} 67,2017$, p. 71-94.

22. Kevin Brookes, Diffusion et transformation $d u$ néo-libéralisme en France des années 1960 aux années 1980 L'Association pour la Liberté et le Progrès Social et les "Nouveaux Économistes", op. cit.

23. Entretien avec Jacques Garello, 8 juin 2012.

24. Lettre de Robert Poole Jr (Reason) à Albert Garand, 24 juin 1978, Archives de l'ALEPS, pochette $\mathrm{n}^{\circ} 2$.

25. Liberté économique et progrès social, $\mathrm{n}^{\circ} 40$, décembre-janvier 1981 (spécial prix Nobel), p.2.

26. Cf. le compte rendu qu'en fait l'ALEPS : « Milton Friedman invité de l'ALEPS le 18 octobre $1980 »$, Liberté économique et progrès social, $\mathrm{n}^{\circ} 40$, décembrejanvier 1981 (spécial prix Nobel), p. 5-16.

27. Je remercie Daniel Steinmetz-Jenkins de nous avoir fourni cette information. Cf. Pascal Salin à Raymond Aron, 4 décembre 1980. Fonds Raymond.
Dossier 94 /4, Bibliothèque Nationale.

28. Fondée en 1955 par Antony Fisher, alors entrepreneur dans l'industrie d'élevage de poulet, ce think tank a acquis sa notoriété par sa production de notes et d'ouvrages académiques et par sa proximité avec Margaret Thatcher. Celle-ci les remerciera peu après son élection pour leur rôle exercé en amont pour préparer l'opinion publique aux réformes dont elle faisait la promotion. Cf. Richard Cockett, Thinking the unthinkable, op. cit. 29. Jean-Jacques Rosa à IEA, 15 février 1977 ; John Wood à Jean-Claude Casanova, 25 février 1976, Archives de l'Institute for Economic Affairs, Box 126, Hoover Institution Archives.

30. John Wood à Michel Drancourt, 31 janvier 1977, Archives de l'Institute for Economic Affairs, Box 126, Hoover Institution Archives.

31. Ibid.

32. Cette organisation internationale est fondée en 1981 par le fondateur de l'Institute of Economic Affairs, Antony Fisher pour propager ce modèle dans le monde entier en soutenant la création et le développement de think tanks acquis aux idées libérales et conservatrices. Sur son rôle dans la promotion du modèle des think tanks dans le monde voir Marie-Laure Salles-Djelic, "Building an architecture for political influence: Atlas and the transnational institutionalization of the neoliberal think tank", in Christina Garsten et Adrienne Sörbom (dir.), Building an architecture for political influence: Atlas and the transnational institutionalization of the neoliberal think tank: Corporate Engagement in Politics and Governance, Edward Elgar Publishing, 2017, p. 25-44.

33. Léonard Liggio, « D’où vient la réussite de Ronald Reagan. Ideas have always consequences. ", Liberté économique et progrès social, $\mathrm{n}^{\circ} 46-47$, Octobre 1983 (numéro double).

34. Entretien avec Guy Plunier, 4 novembre 2016.

35. Brochure de présentation de l'Institut Économique 
de Paris, 1982.

36. «L'institut Économique de Paris », Archives de l'Institute of Economic Affairs. Hoover Institution, box 282 .

37. La littérature académique étant quasiment inexistante sur le sujet, nous renvoyons à la biographie d'Antony Fisher écrite par un membre de l'IEA. Gerald Frost, Antony Fisher: Champion of Liberty, London, Profile, 2002. ; Marie-Laure Salles-Djelic, "Building an architecture for political influence: Atlas and the transnational institutionalization of the neoliberal think tank", op. cit.

38. « Comptes de l'Institut Économique de Paris », mars 1987. Nous remercions Guy Plunier de nous avoir transmis ce document.

39. Lettre John Wood à Florin Aftalion, 25 février 1983, Archives de l'Institute of Economic Affairs. Hoover Institution, box 282.

40. Des livres contre la participation obligatoire dans les entreprises, contre la loi Quilliot, contre le prix unique du livre etc. Christian Fauvelais, Jean-Yves Glain, Le prix unique pour le livre : enquête sur une loi au-dessus de tout soupçon, Éditions de l'Institut Économique de Paris, 1983 ; Pascal Salin, Le piège de la participation obligatoire dans les entreprises, Éditions de l'Institut Économique de Paris, 1984 ; Henri Lepage, Christian Attias, François Guillaumat, Les Vraies clefs de la location: la loi dite Quilliot, Paris, Éditions de l'Institut Économique de Paris, 1984. 41. Catherine Rault, Les Clubs politiques d'opposition entre 1981 et 1986: l'exemple du Club de l'Horloge, mémoire de DEA en science politique, IEP de Paris, 1987.

42. Il amène notamment l'économiste Pascal Salin à réfléchir à en mettre une en place dans sa circonscription de Redon. Cf. François Grosrichard, « En toute franchise », Le Monde, 6 décembre 1985. L'Institut Économique de Paris organise un colloque aux Antilles pour envisager la mise en place d'une zone franche. Cf. «Fin de la deuxième conférence internationale sur les zones franches... Et maintenant ? Il faut imaginer », France-Antilles, 29 mai 1986.

43. Nous nous basons sur les cartons d'invitation aux événements. Archives de la Société du Mont Pèlerin, Hoover Institution Archives, Box 4.

44. Catherine Rault, Les Clubs politiques d'opposition entre 1981 et 1986, op. cit.

45. «Catalogue. Diffusion livres étrangers, $3^{\mathrm{e}}$ trimestre 1985 », Éditions de l'Institut Économique de Paris, Archives de l'Institute of Economic Affairs. Hoover Institution, box 282 .

46. Entretien avec Guy Plunier, 4 novembre 2016.

47. Alain Madelin, «Avez-vous lu Mises ?», Le Figaro Magazine, 9 mars 1985.

48. "Invitation au colloque du 19 février 1986 sur "La privatisation, un défi pour l'Europe" », Archives de la Société du Mont Pèlerin. Hoover Institution Archives, Box 4.

49. Entretien avec Guy Plunier, 4 novembre 2016.

50. Correspondance entre Guy Plunier et Edwin Feulner, « Mont Pèlerin Society records », Hoover Institution Archives, Box n ${ }^{\circ} 78$.

51. Programme de la conférence régionale « l'ordre économique mondial », Friedrich Hayek Papers. Hoover Institution Archives, box 83.

52. Traduit de l'anglais : "I consider as a significant fact that Jacques Chirac has expressed that desire. My own impression is that Jacques Chirac is more and more influenced by sound liberal ideas ", Lettre de Pascal Salin à Friedrich Hayek, le 18 janvier 1984, Hoover Institution Archives. Friedrich Hayek Papers, Box 83.

53. Entretien avec Guy Plunier, 4 novembre 2016.

54. « Allocution prononcée par Monsieur Jacques Chirac, Maire de Paris, à l'occasion de la conférence régionale du Mont Pèlerin et de la remise de la grande 
médaille de Vermeil de la ville de Paris au Professeur Friedrich A. Hayek prix Nobel de Sciences économiques ", " Mont Pèlerin Society records ", Hoover Institution Archives, Box $n^{\circ} 78$.

55. « Friedrich Hayek, Le libéralisme classique est devenu la nouvelle pensée », Le Figaro Magazine, 10 mars 1984.

56. «Mont Pelerin Society's Paris meeting: The world economic order », The Mont Pelerin Society Newsletter, juin 1985.

57. « Paris, capitale du libéralisme ! », Le Figaro Magazine, 10 mars 1984.

58. Christian Descamps, "Von Hayek, gourou du libéralisme ultra », Le Monde, 11 mars 1984.

59. Jacques Julliard, « La nouvelle idole de la droite», Le Nouvel Observateur, 4 juin 1984.

60. Prospectus, « Cap sur le libéralisme. Pour réfléchir ensemble », 14-18 novembre 1985, Archives de Friedrich Hayek. Hoover Institution Archive, Box 26. 61. « Journal de Bord. Cap sur le libéralisme. Pour Réfléchir ensemble. Des hommes pour entreprendre et réussir ", Archives Privées de Serge Schweitzer.

62. Guy Porte, « L'"Eugenio-Costa", bateau-école pour intellectuels », Le Monde, le 20 novembre 1985. 63. Milton Friedman et Rose D. Friedman, La tyrannie du statu quo, Paris, J.-C. Lattès, 1984.

64. Entretien avec René de Laportalière, 23 octobre 2016 ; René de Laportalière, «Libéralisme. Les propositions de Printemps $86 »$, L'Express, 17 mai 1985. 65. Sophie Huet, " "Printemps 86" : les travaux pratiques de l'opposition », Le Figaro, 5 août 1986 ; Guilemette de Sairigné, "Le tondeur de roses », L'Express, 7 févier 1986.

66. René de Laportalière, « Libéralisme. Les propositions de Printemps 86 », L'Express, 17 mai 1985.

67. Entretien avec Gérard Longuet, 6 décembre 2016. 68. James G McGann et R. Kent Weaver, Think Tanks and Civil Societies : Catalysts for Ideas and Action,
New Brunswick, États-Unis, Transaction Publishers, 2000.

69. Rassemblement pour la République et Union pour la démocratie française, Plate-forme pour gouverner ensemble, Paris, France, RPR : UDF, 1986, p. 17.

70. Jérôme Perrier, «La parenthèse libérale de la droite française des années 1980. Le phénomène politique de la « bande à Léo » ou l'échec de la promotion d'un libéralisme contre 1'État », Histoire@Politique, n² 25, 2015, p. 176-196.

71. Kevin Brookes et Benjamin Le Pendeven, « L’État innovant (1)», op. cit.

72. John L. Campbell et Ove K. Pedersen, The National Origins of Policy Ideas, op. cit.

73. Geoffrey Geueens (dir.), « Avant-propos », op. cit. 74. Le Journal des Républicains, ${ }^{\circ} 5$, octobrenovembre 1985 ; Guy Plunier a témoigné de ses rencontres avec Bernard de la Rochefoucault : «Bertrand de la Rochefoucauld, de temps en temps, m'invitait à déjeuner pour savoir...il se demandait comment je faisais pour.... L'Institut Économique de Paris, avec son système de travail et de diffusion », entretien avec Guy Plunier 4 novembre 2016.

75. François Krug, « Agnès Verdier-Molinié. Égérie de la diète publique », Le Monde, 27 janvier 2017.

76. Ibid.

77. Benjamin Tainturier, Pour la liberté e-limitée, Internet et les institutions du libéralisme en France, mémoire de M2, École Normale Supérieure, Paris, 2017.

78. Frank Dedieu, « Les Nouveaux libéraux en embuscade », L'Expansion, 3 octobre 2015.

79. Gaspard Koenig de GénérationLibre, Mathieu Laine d'Altermind, Cécile Philippe de l'Institut Economique Molinari, Agnès Verdier-Molinié de la Fondation iFRAP, sont régulièrement les invités des plateaux de télévision.

80. Stephen Boucher et Martine Royo, Les think tanks, 
op. cit., p. 34.

81. Cf. Sébastien Caré, Les libertariens aux ÉtatsUnis. Sociologie d'un mouvement a-social, Presses Universitaires de Rennes, 2010 ; Seymour Martin Lipset et Asoke Basu, « Des types d'intellectuels et de leurs rôles politiques », Sociologie et sociétés, vol 1, 1975, p. 50-90..

82. C'est ce que l'étude comparée de John Campbell et Ove Perdersen fait transparaître. John L. Campbell et Ove K. Pedersen, The National Origins of Policy Ideas, op. cit.

83. James McGann, « 2017 Global Go To Think Tank Index Report », TTCSP Global Go To Think Tank Index Reports, 31 janvier 2018.

\section{$\mathrm{R} \cdot \dot{E} \cdot \mathrm{S} \cdot \mathrm{U} \cdot \mathrm{M} \cdot \dot{E}$}

Les think tanks, organisations à la croisée des savoirs et du pouvoir, constituent une forme d'organisation privilégiée par les militants néo-libéraux dans le monde. À partir d'un travail sur les archives d'organisations internationales et de l'analyse d'entretiens, cet article montre comment certains réseaux internationaux ont encouragé la création de think tanks au début des années 1980 en France. L'objectif d'organisations comme l'Institut économique de Paris ou Printemps 86 était d'influencer le débat public et de transformer le discours économique des droites qui étaient alors dans l'opposition. Leurs répertoires d'action, structure et financement sont directement inspirés par ceux des think tanks anglais et américains. Cependant, ces organisations ont eu des difficultés à s'institutionnaliser avant qu'une deuxième vague de création de think tanks au début des années 2000 revitalise la promotion de ces idées en leur assurant une certaine médiatisation.

\begin{abstract}
Think tanks, organizations at the crossroads of knowledge and power, are a form of organization favored by neo-liberal advocates around the world. Based on work on archives of international organizations and analysis of interviews, this article shows how some international networks encouraged the creation of think tanks in the early 1980s in France. The goal of organizations like the Institut économique de Paris or Printemps 86 was to influence the public debate and transform the economic discourse of the right wing political parties that were then in the opposition. Their repertoires of action, structure and funding are directly inspired by those of the British and American think tanks. However, these organizations struggled to become institutionalized before early 2000 s revitalized the promotion
\end{abstract}


of these ideas by giving them some media coverage. 
